THE ROLE OF PLATELETS AND MAST CELLS IN HELICOBACTER PYLORI-INDUCED CHANGES IN RAT GASTRIC MUCOSAL MICROCIRCULATION IN VIVO. N. Kalia, D. Morton', S. Jacob, N.J. Brown*, M.W.R. Reed*, K.D. Bardhan ${ }^{\circ}$, Depts. of Biomedical Science and Surgery*, University of Sheffield, S10 2TN; 'Rotherham District General Hospital NHS Trust, Rotherham, S60 2UD.

Background/Aims: The mechanisms by which Helicobacter pylori $(H$. pylori) infection results in gastric mucosal injury are unknown. This study aimed to assess i) whether $H$. pylori extracts could initiate an inflammatory response in vivo in the rat gastric mucosal microcirculation ii) a possible role for mucosal mast cells.

Methods: The gastric mucosa of anaesthetised rats was continuously superfused with extracts of $H$. pylori, Escherichia coli (E. coli) or distilled water (controls). Fluorescent in vivo microscopy was uséd to assess macromolecular leakage of fluorescein labelled albumin from mucosal vessels and to study leukocyte and platelet activity over 90 minutes. A separate group of animals were orally administered ketotifen, a mast cell stabiliser $(100 \mu \mathrm{g} / 100 \mathrm{~g}$ bwt), prior to anaesthesia and subsequent $H$. pylori treatment.

Results: $H$. pylori extracts induced albumin leakage with a peak of $11 \%$ at 5 minutes $(P<0.02)$. There was significant increases in adherent platelet thrombi peaking at 15 minutes $(P<0.001)$ and plateauing at this level and also circulating platelet emboli with a peak at 30 minutes $(P<0.001)$. Although a significant increase $(P<0.001)$ in the number of rolling leukocytes was seen (peak at 5 minutes), they did not become fully activated to adhere. A second topical application of $H$. pylori had no effect. These events were unique to $H$. pylori. Ketotifen pre- treatment prevented macromolecular leakage but did not prevent platelet aggregation.

Conclusion: This is the first study in vivo to demonstrate the ability of H. pylori extracts to promote an increase in microvessel permeability and platelet aggregation within gastric mucosal microvessels. Since ketotifen prevented only macromolecular leakage, separate mechanisms may be involved in $\mathrm{H}$. pylori-induced microcirculatory changes.

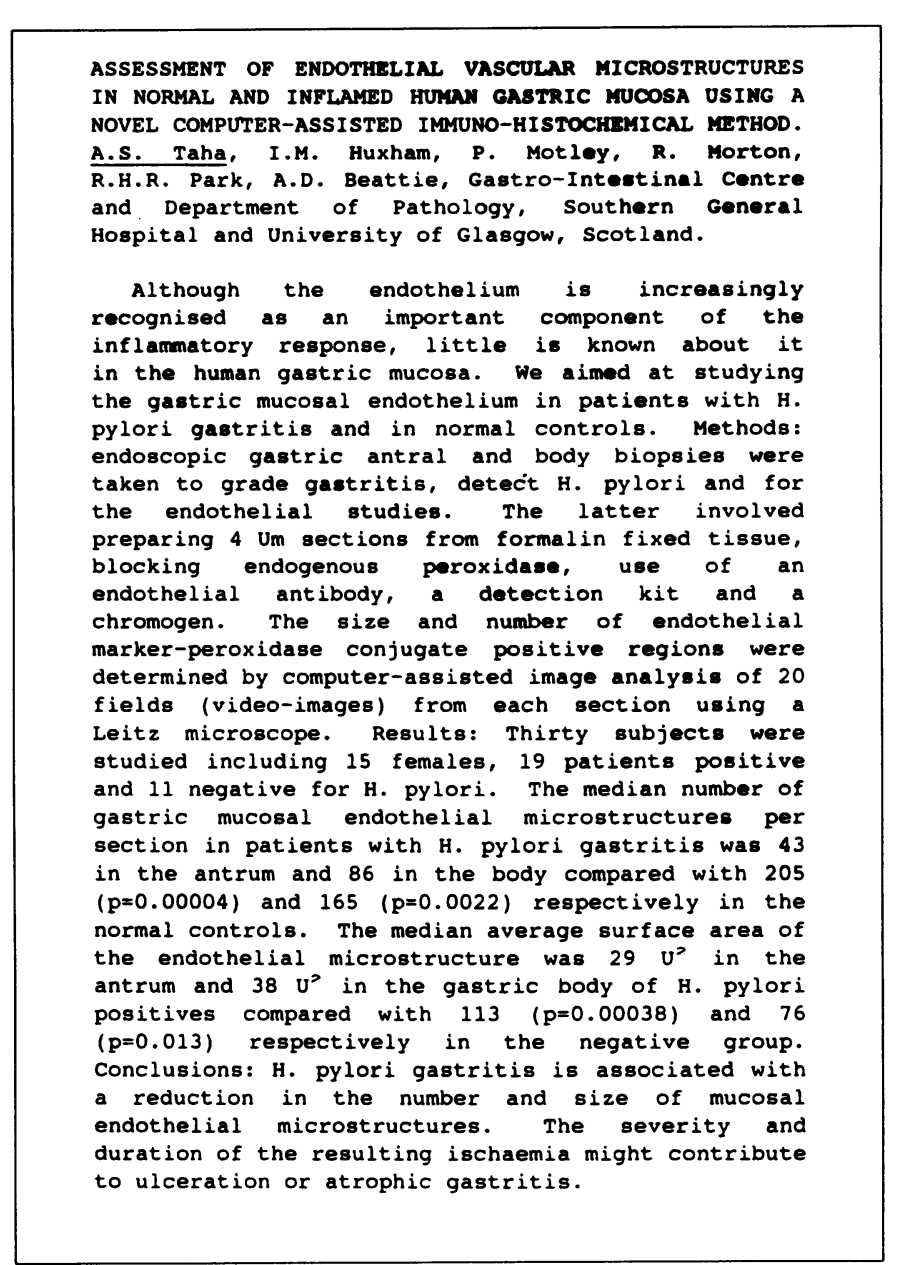

\section{Ulcers and therapy T152-T157}

T152

RELEVANCE OF ANTIBIOTIC SENSITIVITIES IN PREDICTING FAILURE OF OMEPRAZOLE, CLARITHROMYCIN AND TINIDAZOLE TO ERADICATE HELICOBACTER PYLORI. $\underline{\mathrm{P}}$ Moayyedi, DS Tompkins*, N Mapstone, ATR Axon. Centre for Digestive Diseases, Leeds General Infirmary, *Dept. Public Health Medicine, Leeds

Introduction: Omeprazole $250 \mathrm{mg}$ od/bd, clarithromycin $250 \mathrm{mg}$ bd and tinidazole $500 \mathrm{mg}$ bd for 7 days (OCT) is an effective regimen against $H$ pylori but it is unclear whether antibiotic resistance reduces the efficacy of this treatment.

Methods: $H$ pylori was cultured from antral biopsies of infected patients as determined by histology, rapid urease test and ${ }^{13} \mathrm{Carbon}$ Urea Breath Test $\left({ }^{13} \mathrm{C}\right.$-UBT). Patients were prescribed $\mathrm{OCT}$ and cure of the infection was assessed by ${ }^{13} \mathrm{C}$-UBT at least 4 weeks after completion of therapy: Antibiotic sensitivities of $\mathrm{H}$ pylori to 5 nitroimidazoles and macrolides was evaluated using the disc diffusion method using $5 \mu \mathrm{g}$ metronidazole and a $15 \mu \mathrm{g}$ clarithromycin on blood agar plates incubated microaerobically for 72 hours.

Results: $141 \mathrm{H}$ pylori infected patients were enrolled in the study and the organism was successfully cultured in 119 cases (84\%). The incidence of metronidazole resistant strains was $49 / 119$ (41\%), clarithromycin resistance $5 / 119(4.2 \%)$ and dual antibiotic resistance $4 / 119(3.4 \%)$. The overall eradication rate was $125 / 141(89 \%)$. OCT was successful in $62 / 69(90 \%)$ patients harbouring fully sensitive strains of $H$ pylori compared with $42 / 45(93 \%)$ of patients with strains that were resistant to metronidazole alone ( $p=1.0$ Fisher's exact test). The eradication rate in those harbouring $H$ pylori strains resistant to both antibiotics was $1 / 4(25 \%)(p=0.003$ Fisher's exact test) whilst treatment was successful in the one patient with $H$ pylori resistant to clarithromycin alone. Repeat culture in treatment failures revealed dual antibiotic resistance in 9 , metronidazole resistance in 4 and fully sensitive organisms in 3 cases.

Conclusion: The efficacy of OCT is not reduced in 5-nitroimidazole resistant $H$ pylori strains but dual antibiotic resistance may be associated with treatment failure. Secondary metronidazole and clarithromycin resistance is common in unsuccessfully treated cases. treated rats was $0.14 \pm 0.02$ (mean and SEM) versus $0.22 \pm 0.04$ in the controls ( $p<0.025)$. The same pattern was seen in the corpus: $0.13 \pm 0.02$ (mean and SEM) in the iodoacet
versus $0.20 \pm 0.02$ in the controls $(p<0.02$ )

Conclusion. lodoacetamide induces inflammation in the gastric mucosa resulting in a reduction in SST mRNA. This suggests that the inflammation occuring in $\mathrm{Hp}$ infection may be the primary cause of the alteration in expression of gastrin and SST. 


\section{A RANDOMUSED TRAL OF OPENACCESS GASTROSCOPY VERSUS EMPIRICAL TREATII ENT FOR DYSPEPSU WN PATIENTS UNDER 45 OTode PA Lombard MG \& Settion GP. Study Group \\ Aintree Hospitals (NHS) Trust \& Gestroenterology Research Unit, Dept of Medicine, University of Liverpool.}

INTRODUCTion Provision of open-access gastroscopy (OAG) has expanded rapidly in recent years but its place in the investigation of younger patients with dyspepsia is still debated. Some argue that early endoscopic assessment may allow more rational prescribing and thereby reduce costs. Others suggest that investigation should be reserved for patients who fail to respond to an empirical course of acid-suppressive therapy. Ams To assess the costeffectiveness and clinical benefits of these two approaches. Menmops Patients under the age of 45 presenting to participating GPs with dyspepsia were randomised to either an 8-week course of ranitidine (group A), or an OAG, performed within 48 hours of referral (group B). Management of group B patients after gastroscopy, and group A patients after the initial 8-week treatment period, was entirely at the discretion of the GPs. Symptomatic response in the two groups was assessed at 3-month intervals for one year and the costs of all dyspepsia-related consultations, investigations and medication (including "over the counter" antacids/alginates) were compared. RESULTS 69 patients were recruited; 33 in group A and 36 in group B. $72 \%$ of group $B$ had positive findings on endoscopy. Only 6 patients in group $A$ went on to have a gastroscopy. Overall compliance with follow-up was $73 \%$ but cost data was obtained in all patients trom GP records. Symptomatic response was slightly better in the OAG group at 4 weeks but there were no significant differences in mean symptom score between the wo groups throughout the rest of the year. Cost analysis showed empirical ranitidine treatment to be the more cost-effective strategy. Excluding the cost of initial treatment (OAG or ranitidine), subsequent management was more expensive in group $B$ because of increased prescription costs for those with positive findings at endoscopy. This out-weighed the savings from reduced prescribing in patients with normal gastroscopies. The number of dyspepsia-related consultations and gastrointestinal investigations other than gastroscopy were similar in the two groups. ConcLusions in young patients with dyspepsia, OAG before treatment results in greater costs without obvious benefits in terms of symptom control.
OPEN ACCESS GASTROSCOPY FINDINGS ARE UNRELATED TO USE OF ASPIRIN AND NSAIDS

I.C.Mansfield. J.R.Greenaway. N.L.Idle. M.G.Bramble.

Gastroenterolog! Unit. South Cleveland Hospital, Middlesbrough.

The incidence of serious GI side effects from NSAIDs and aspirin is known to be low from epidemiological studies. Dyspepsia is common and is increasingly investigated by general practitioners (GPs) using open access gastroscopy (OAG). The aim of this study was to determine whether NSAID and / or aspirin usage was associated with different endoscopic findings in the dyspeptic patients referred for OAG.

OAG results for the last six years were reviewed. A total of 8135 patients were gastroscoped. all of whom had symptoms. On the referral forms GPs indicated whether the patients were taking NSAIDs or aspirin, 759 were on NSAIDs and 281 on aspirin.

Patients taking NSAIDs and / or aspirin were generally older, $43.4 \%$ being over the age of 60 . compared with $23.5 \%$ over 60 not on these drugs $(p<0.01)$. The major endoscopy findings were dependent on age. The incidence of gastric ulcers rose from $2.1 \%$ below' 60 years to $4.8 \%$ above $(p<0.001)$. The frequency of oesophagitis also rose slightly $18.3 \%$ vs $22.7 \%$. but duodenal ulcers were unchanged at $12 \%$. Patients taking NSAIDs and / or aspirin had no significant differences in frequency of these diagnoses when age matched groups were compared. For example patients over 60 years on NSAIDs or aspirin had a $5.6 \%$ frequency of gastric ulcer vs $4.8 \%$ in those not on NSAIDs / aspirin ( $p>0.5$ ).

In conclusion. although NSAIDs and aspirin are frequently implicated in GI bleeding in the elderly, in patients referred for investigation of dyspepsia there is no increase in major endoscopic pathology. The incidence of gastric ulceration rises with age regardless of NSAID / aspirin usage. These results indicate that age, but not NSAID or aspirin usage, should be a factor in selecting which dyspeptic patients to refer for OAG.
BENEFTSS OF OMIGPRAZOLE OVER MISOPROSTOL IN HEALING NSAID-ASSOCLATED ULCERS CI Hawkey' ${ }^{1}$ A Swannel12 ${ }^{2}$, Eriksson ${ }^{3}$, A Walan ${ }^{3}$, I Lofberg ${ }^{3}$, E Taure ${ }^{3}$, I Wiklund ${ }^{3}$, ND Yeomans4. 'Div Gastro, Univ Hosp, ${ }^{2}$ Dept of Rheuma/Rehab, City Hosp, Nottingham UK. ${ }^{3}$ Astra Hassle AB, Sweden. 'Dept of Med, Western Hosp, Melbourne, Australia.

BACKGROUND Ulcer and erosions are commonly associated with nonsteroidal anti-inflammatory drugs (NSAIDs), but there are few comparative data to help identify optimal trearment. AM To compare omeprazole (ome) and misoprostol (miso) for healing of gastroduodenal lesions (ulcers/multiple erosions), incidence of adverse events and influence on Quality of Life in patients on continuous NSAID therapy.

METHODS International multicenter study with a double-blind parallelgroup design. Patients with ulcers $(>3 \mathrm{~mm})$ and/or more than 10 erosions in either stomach or duodenum were randomised to ome $20 \mathrm{mg}$ once daily, ome 40mg once daily or miso $200 \mu \mathrm{g}$ four times daily and treated for 4 or 8 weeks. Healing was defined as healed ulcers, $<5$ erosions in stomach and $<5$ erosions in duodenum, and not more than mild dyspeptic symptoms. Quality of Life was assessed using questionnaires, including The Gastrointestinal Symptom Rating Scale (GSRS). RESULTS A total of 935 patients were enrolled into the study and 922 were evaluable in an All Patients Treated analysis. There was no significant difference in overall healing between the treatment groups (Mantel-Haenszel life-table test). Cumulattre healing rates (healing as defined above) All patients

\begin{tabular}{lllllll} 
& \multicolumn{2}{c}{ ome20mg } & ome40mg & miso & \multicolumn{3}{c}{ ome20mg } & ome40mg & miso \\
$\mathrm{n}=$ & 308 & 316 & 298 & 189 & 204 & 200 \\
4 wks & $56 \%$ & $60 \%$ & $56 \%$ & $57 \%$ & $62 \%$ & $52 \%$ \\
8 wks & $76 \%$ & $75 \%$ & $71 \%$ & $78 \%$ & $73 \%$ & $67 \%$
\end{tabular}
In patients with ulcer at entry, the healing rates were higher for ome than for miso but not statistically significant. Fewer patients discontinued due to lack of efficacy on ome (ome $20 \mathrm{mg} 4 \%, p=0.01$; ome $40 \mathrm{mg} 3 \%$, $\mathrm{p}=0.002$ ) than on miso $(9 \%)$. GSRS scores showed less diarrhoea on both doses of ome $(p<0.002)$ than on miso and less abdominal pain for ome $20 \mathrm{mg}(p=0.008)$ than on miso. CONCLUSION Omeprazole and misoprostol are effective treatments for NSAID-associated gastroduodenal lesions. Omeprazole is associated with higher ulcer healing rates and fewer gastrointestinal adverse events than misoprostol.
Endoscopic injection for bleeding peptic ulcer: a comparison of Adrenaline alone with Adrenaline plus human Thrombin

Kubba AX, Murphy W \& Palmer K R

GI Unit, Western General Hospital, Edinburgh, Scotland .

Endoscopic irjection therapy with dilute adrenaline improves outcome in bleeding peptic ulcer , but $15-24 \%$ of patients rebleed despite optimum injection. Human thrombin may more effectively thrombose the bleeding artery and stop bleeding , but risks local and systemic complications .

One hundred and forty patients with significant peptic ulcer haemorrhage and endoscopic stigmata of recent haemorrhage were randomised to endoscopic injection with 4-18 ml of 1:100 000 adrenaline (70 patients, group 1) or to adrenaline plus 600-1000 IU of human thrombin dissolved in $40 \mathrm{MM}$ of calcium chloride $(70$ patients, group 2). The two groups were well matched for age, shock, haemoglobin concentration, endoscopic findings and consumption of non-steroidal antiinflammatory drugs .

No complications of therapy occurred . Fourteen (20\%) group 1 patients and three (4.5\%) group 2 patients rebled $(p<0.005)$. The 30 day mortality was $10 \%$ in group 1 patients and nil in group 2 patients $(p<0.007)$. Transfusion requirements and hospital stay were similar in the two groups.

Human thrombin injection appears safe and may represent best treatment for bleeding peptic ulcers. 
HAEMOSTASIS OF PEPTIC LESIONS USING MINIMALLY INVASIVE INTRAGASTRIC SURGERY: AN EXPERIMENTAL MODEL

C.C.Hepworth, S.S.Kadirkamanathan, F.Gong, C.P.Swain, J.Rogers. GI Science Research Unit, St Bartholomew's and The Royal London School of Medicine and Dentistry, London, UK.

Bleeding peptic ulcer can have a high mortality in elderly patients who often have concomitant illness and who undergo conventional surgery. A less invasive surgical method for oversewing bleeding ulcers might be safer. We have developed new devices and methods to assist with such a minimally invasive technique. This technique relies upon the placement of three gastrostomy tubes (one $10 \mathrm{~mm}$ and two $5 \mathrm{~mm}$ internal diameter tubes), delivered by the standard 'push pull' technique. The gastrostomy tubes used are tapered up to the required internal diameter and have a flange on their distal end to prevent dislodgement from the stomach. Standard laparoscopic ports can then be placed onto these gastrostomies, enabling laparoscopic equipment to be used. This technique allows direct access to the entire of the stomach and might have the added advantage of being performed under a local anaesthetic and sedation. This technique has been performed in both post mortem tissue $(\mathrm{n}=10)$ and in animal models $(n=4)$ under general anaesthetic. Artificial ulcers $(n=10)$ have been created and complete haemostasis achieved quickly by oversewing with needle and thread using laparoscopic equipment. Closure of gastrostomies is performed by passage of 2 threads (one either side of the gastrostomy) via small cannulae into the lumen of the stomach. By means of a gastroscope the threads can be passed to forceps introduced down the gastrostomy. Threads are then withdrawn and tied extracorporeally and replaced into the stomach This procedure can be repeated, until sufficient threads $(6-10)$ have been passed to close the gastrostomy hole. The gastrostomy is then removed with a gastroscope, the threads tied submucosally and the skin closed. Survival experiments $(n=3)$ demonstrate that gastrostomies can be closed (with antibiotic cover) in this manner and no infection or fistula formation has occurred. Animals are eating the following day. Conclusion 1) Haemostasis of bleeding lesions is possible using this minimally invasive technique. 2) Closure of the gastrostomy can be performed safely and easily if required. Further studies are required to assess the suitability of this form of surgery in man, in particular its application under sedation and local anaesthetic.
BACTERIAL TRANSLOCATION IS ASSOCLATED WITH UPPER GASTRONNTESTINAL COLONIZATION IN SURGICAL PATIENTS O'Boyle CJ, Murchan P, Mitchell CJ, Macfie J.

Combined Gastroenterology Unit, Scarborough General Hospital, Scarborough, North Yorkshire.

Alteration in gastrointestinal microflora may predispose to bacterial translocation. The aim of this study was to relate changes in upper gastrointestinal flora with the occurrence of bacterial translocation and to determine whether or not this was associated with any increase of septic morbidity.

Upper intestinal flora was assessed preoperatively by culturing aspirates from indwelling nasogastric suction tubes. At subsequent laparotomy bacterial translocation was determined by biopsy and culture of mesenteric lymph nodes. All postoperative septic complications were carefully recorded.

A total of 136 patients was studied. There was a lower incidence of septic complications in patients with sterile aspirates $(31 \%$ vs $64 \%$, $p=0.035 \chi^{2}=4.17$ ). Translocation of bacteria occurred more commonly in patients who grew multiple organisms from their aspirates than in those growing mono-or no organisms ( $48 \%$ vs $16 \%$ ) $\left(p=0.01, \chi^{2}=6.64\right)$. Although bacterial translocation was associated with an increased incidence of subsequent septic complications $(16 / 29,55 \%$ vs, $30 / 10728 \%)$ this was not significant.

We conclude that bacterial translocation is related to overgrowth of upper gastrointestinal tract microflora and is associated with increased postoperative septic complications in surgical patients.

\section{Nutrition T158-T163}

OPTIMISATION OF GLUCOSE POLYMER CHAIN LENGTH IN ORAL REHYDRATION SOLUTION USING A HUMAN CHOLERA MODEL. IL Turvill, FH Mourad, MJG Farthing. Digestive Disease Research Centre, St Bartholomew's \& The Royal London School of Medicine \& Dentistry, London.

Introduction. Recent studies indicate that complex carbohydratebased oral rehydration solution (ORS) are more effective in promoting water absorption than glucose monomer-based ORS. Naturally occurring complex carbohydrates (eg rice starch) are relatively insoluble in water and difficult to prepare and package. Shorter chain glucose polymers are more soluble in water, easily packaged and may enhance water absorption as effectively as complex carbohydrates. We have already found in rat small intestine exposed to cholera toxin (CT) that a 50 chain length glucose polymer-based ORS (glucidex 2) enhanced more water absorption than shorter chain length glucose polymers.

Aim. We measured water and electrolyte absorption in a human cholera model using ORS containing (I) glucidex 2, (ii) glucose monomer and (iii) 5 chain length glucose polymer (glucidex 21).

Methods. A triple-lumen perfusion tube was positioned in the jejunum under fluoroscopy. A $30 \mathrm{~cm}$ closed segment was isolated between two inflatable balloons on the tube into which $20 \mu \mathrm{g}$ CT was introduced. After $3 \mathrm{~h}$ incubation, the different ORS were perfused in a random order. ORS composition: $\mathrm{Na} 60, \mathrm{~K} \mathrm{20}, \mathrm{Cl} 60$, citrate 10 and $90 \mathrm{mmol} / \mathrm{L}$ glucose or glucose 5 or 50 chain length polymer equivalent. A tracer of a non absorbable marker $\left(\left[{ }^{14} \mathrm{C}\right]-\mathrm{PEG}\right)$ was used to assess net water movement.

Results. All three solutions induced net water absorption (glucose: med $2.26 \mathrm{ml} / \mathrm{cm} / \mathrm{h}$ [IQR 2.89 to 1.72 ], $\mathrm{n}=6 ; \mathrm{G} 5: 5.39$ [10.02 to 3.66], $\mathrm{n}=5$; $\mathrm{G50}$ : 6.19 [9.49 to 3.85$], \mathrm{n}=7$.). Both $\mathrm{G5}$ and $\mathrm{G} 50$ polymers significantly enhanced absorption compared to glucose $(p<0.02)$. There was a significant positive correlation between water absorption and increasing glucose polymer chain length (Cuzick's test for trend $p<0.02$ )

Conclusion. These preliminary results support our previous findings in animal experiments suggesting that the optimal glucose polymer chain length is $\mathbf{5 0}$ glucose units.

\section{IMMEDIATE FEEDING AFTER PERCUTANEOUS ENDOSCOPIC GASTROSTOMY (PEG) INSERTION. IS IT SAFE?}

TD Heymann, K Chopra, K Hills, P Nield, D Westaby. Department of Gastroenterology, Charing Cross and Chelsea \& Westminster Hospitals, London UK.

PEG formation is associated with a small but important risk of early complication including peritoneal leakage and pulmonary aspiration. A $12 \mathrm{hr}$ delay of feeding has been widely adopted to minimise the risk. However, combined with the pre-procedural fast, there may be 24 hours without nutrition. This is undesirable in patients with existing nutritional deficit.

In the present randomised study, the safety of immediate feeding IF) was compared to a delay of $6 \mathrm{hrs}$ to start sterile water and 12 hours to feeding (DF). Information sheets were provided for the attending staff who initiated feeding, documented reasons for delay and adverse events. IF was sanctioned by the endoscopist based upon an uncomplicated procedure. Patients in the DF group were assessed for complications by the attending medical staff at $6 \mathrm{hrs}$ and $12 \mathrm{hrs}$ prior to commencing water and then feed.

52 consecutive patients were randomised, 25 to IF and 27 DF. IF group feeding was commenced at a median of 2 hrs(range 1-5) post PEG insertion compared to 17 hours(12-50) in the DF group. The excess delay in the DF group was attributed to unsanctioned deferement when feeding was scheduled beween $22.00-0600 \mathrm{hrs}$. Six patients (12\%) developed major complications (IF\&DF 3). In one (DF) intraperitoneal bleeding required laparotomy. The remaining 5 patients required cessation of tube feeding for a median of 2 days (range 1-10) as a consequence of tube site pain (2IF), peritonism (2DF) and gastric mucosal bleeding (IDF).

This study highlights the significant early complication rate associated with PEG formation which may cause delay in tube feeding. The early introduction of feeding did not increase the risk and minimised the nutritional deprivation that accompanies PEG insertion. 